\title{
Management of primary pterygium with intra-lesional injection of 5 flurouracil and bevacizumab (Avastin)
}

\author{
Noha Ghoz ${ }^{1}$. John Britton ${ }^{1} \cdot$ Andrew R. Ross ${ }^{1} \cdot$ Imran Mohammed $^{1} \cdot$ Emily Hogan $^{1} \cdot$ Dalia G. Said $^{1}$. \\ Harminder S. Dua ${ }^{1}$
}

Received: 8 December 2018 / Revised: 29 April 2019 / Accepted: 10 May 2019 / Published online: 19 June 2019

(c) The Author(s), under exclusive licence to The Royal College of Ophthalmologists 2019

\begin{abstract}
Background To assess the efficacy of combined 5FU and Avastin injections in the treatment of primary pterygium Methods Sixteen eyes with primary pterygium received intralesional 5 fluorouracil and Avastin $(2.5-5 \mathrm{mg})$ injections every 2 weeks for a maximum of five injections. Fourteen eyes of 14 patients received five injections, one eye received three injections and one eye received two injections. All eyes were followed at monthly intervals for 3 months after last injection. Tissue was obtained by surgical excision of primary pterygium from four eyes who received injections and three eyes with primary pterygium who did not receive injections (control) and subjected to immunohistological examination for beta fibroblast growth factor (bFGF), vascular endothelial growth factor (VEGF), epidermal growth factor (EGF), vonWillebrand factor (vWF), lymphatic vessel endothelial hyaluronan receptor (LYVE-1) and collagen-I.

Results Pterygium progression was arrested in all patients. Sixty-two percent of patients had improvement of redness while $89 \%$ had reduced thickness of the lesion. VEGF, bFGF, EGF, vWF, LYVE-1 and collagen-I were all reduced in the injected samples.

Conclusions The injection of 5 fluorouracil and Avastin act synergistically to arrest progression and induce atrophy in primary pterygium. This is related to the effect of agents on fibroblasts, collagen, and vascular tissues. Such medical intervention is a safe and viable option in the management of primary pterygium though excision of residual tissue is still required in some cases. Longer follow up is needed to ascertain whether this will reduce the recurrence rate following excision.
\end{abstract}

\section{Introduction}

Pterygium is an elastotic degeneration of the subconjunctival tissue that affects the exposed bulbar conjunctiva, nasally more than temporally [1]. It affects sight when the fleshy fibrovascular tissue advances across the

These authors contributed equally: Noha Ghoz, John Britton

Supplementary information The online version of this article (https:// doi.org/10.1038/s41433-019-0493-0) contains supplementary material, which is available to authorised users.

Harminder S. Dua

harminder.dua@nottingham.ac.uk

1 Academic section of ophthalmology, Division of Clinical Neuroscience, University of Nottingham and Department of Ophthalmology, Nottingham University Hospitals, NHS Trust, Nottingham, UK limbus and encroaches on to the visual axis of the cornea. It may result in corneal scarring, chronic ocular surface inflammation and irregular astigmatism and can restrict ocular movement [2]. Although the aetiology of the pterygium remains an enigma, environmental factors such as ultraviolet light, dryness, genetic and immunological factors play a big role in its occurrence [3]. Other important factors such as fibrovascular proliferation, inflammation and angiogenesis have been implicated in the pathogenesis and progression of pterygium [4]. Increased expression of several growth factors, such as epidermal growth factor (EGF), vascular endothelial growth factor (VEGF), basic fibroblast growth factor (bFGF), transforming growth factor-beta, connective tissue growth factor, insulin-like growth factor and nerve growth factor has been reported in primary and recurrent pterygia [5]. VEGF, VEGF receptors and other molecules that play a role in angiogenesis are particularly expressed in pterygium [6-8]. 
The gold standard for treatment of primary pterygium is surgical excision with an autoconjunctival graft [9]. Recurrence rates of $0-14.29 \%$ have been reported [10]. Recently Said et al. [11] emphasised the importance of recognising conjunctival recurrence of pterygium and demonstrated that intralesional 5 fluorouracil (5FU) could arrest progression of pterygium avoiding need for surgery. This has also been the experience of others [12]. Studies have also shown that intralesional injection of bevacizumab can treat recurrent pterygium [13, 14].

Taking a cue from the above reports, investigators looked at treating primary pterygium in a similar manner. Two studies were carried out in 2016 to study the effect of intralesional 5FU injection in primary pterygium and reported good results $[12,15]$. Further studies were carried out on the use of Avastin in primary pterygium and also reported good results $[13,16]$.

We conducted a clinical trial to evaluate the effect of combined injections of 5FU and Avastin in patients presenting with primary pterygium. Immunohistology for different growth factors and markers for blood vessels and lymphatics was carried out in tissue samples obtained after excision of injected pterygia. Our results have implications for clinical application of these injections to arrest progression and reduce vascularity of pterygium as the only treatment or prior to surgical excision.

\section{Materials and methods}

The study was approved by the Nottingham Research Ethics Service Committee, East midlands-Nottingham 2; the Medicines \& Healthcare products Regulatory Agency and the Health Research Authority UK. It was registered online and adopted by National Institute for Health Research. Trial registration number is NCT02530801. The study adhered to the tenets of the Declaration of Helsinki. Participants were recruited from specialist cornea and ocular surface clinics at the Queen's Medical Centre, Nottingham University Hospitals NHS Trust. The inclusion criteria were patients with progressive pterygium who sought intervention and had no previous surgical or medical treatment for the condition. Females of childbearing age were excluded unless effective contraception was used.

\section{Outcome measures}

\section{Primary outcome}

Arrest of progression and reduction of vascularity (redness) of lesion.

\section{Secondary outcome}

Cosmetic benefit as perceived by the patient, improvement in symptoms of irritation, reduction in thickness, effect on astigmatism.

All patients were clearly informed that the treatment would not cause the lesion to disappear and that for cosmetic or other reasons surgery to remove the lesion might still be required.

A complete eye examination at base line and after injections was carried. Visual acuity was assessed using LogMAR charts followed by pupil reaction, anterior segment biomicroscopic examination, corneal topography (Oculus Pentacam HR, Oculus Optikgerate GmbH, Wetzlar, Germany) to assess corneal astigmatism, Optical coherence tomography (OCT) (Spectralis Heidelberg engineering, Heidelberg, Germany) at two fixed sites to assess thickness, intraocular pressure measurement and fundal examination. Demographic data were documented. The pterygium length, from limbus to apex was measured with ImageJ software (ImageJ V.1.31, Maryland, USA) before and after injections. Any complications and adverse events were noted and recorded. Anterior segment photographs were taken at every follow-up visit to assess response to treatment in terms of redness and progression. A standardised protocol was followed for both anterior segment photography and OCT. The pterygium was graded as severe (red areas more than white (heavily vascularises with dilated and full vessels and fleshy); moderate (more white areas then red, (intermediate vascularisation with thin and narrow vessels and not fleshy), and mild (atrophic and thin with minimal vasculature). A score of 3,2 and 1 was ascribed to the three grades, respectively. Astigmatic changes were analysed using Vector Analyzer software (Astigmatism Vector Analyzer 1.2, Dr. Peyman) and the magnitude of difference vector was measured.

Injections were administered every 2 weeks for a maximum of five injections or until the desired endpoint was achieved (atrophic pterygium, mild grade). Further follow up was at monthly intervals for 3 months.

\section{Injection method}

All injections were given in the outpatient clinic using a slit lamp. Topical anaesthesia (tetracaine minims $1 \% \mathrm{w} / \mathrm{v}$, Bausch \& Lomb, UK) and povidone iodine $(5 \% \mathrm{w} / \mathrm{v}$, Bausch \& Lomb, UK) eye drops were instilled in the eye $10 \mathrm{~min}$ prior to the injection. With a pre-loaded $1 \mathrm{ml}$ syringe of $5 \mathrm{FU}(0.3 \mathrm{ml}$ of $2.5 \mathrm{mg} 5 \mathrm{FU}$ per $0.1 \mathrm{ml}) 0.1-0.2 \mathrm{ml}$ of $5 \mathrm{FU}(2.5-5 \mathrm{mg})$ was injected into the body of the pterygium using a 30 gauge needle (Sterican, B. Braun, Hessen, Germany). Formation of a bleb indicated intra-lesional injection of drug (Supplementary Fig. 1). This was followed by injection of Avastin, which too 
was delivered in a pre-loaded $1 \mathrm{ml}$ syringe containing $0.2 \mathrm{ml}$ of $5 \mathrm{mg}$ Avastin. $0.1-0.2 \mathrm{ml}$ of Avastin $(2.5-5 \mathrm{mg}$ ) was injected in the body of the pterygium. Post injection, 1-2 drops of chloramphenicol $0.5 \%$ (preservative free minims, Bausch and Lomb, UK) were instilled topically and continued four times a day for 3 days after each injection. All patients had the ability to access the $24 \mathrm{~h}$ on call emergency eye service and contact the investigators for any queries during the study period.

The method deployed was essentially the same as was used for treatment with recurrent pterygium with $5 \mathrm{FU}$ injections only. A total of $0.3 \mathrm{ml}$ of $2.5 \mathrm{mg} / 0.1 \mathrm{ml}(7.5 \mathrm{mg})$ of $5 \mathrm{FU}$ was prepared and dispensed by the pharmacy in a pre-loaded syringe. After preparation of the eye with topical anaesthesia and povidone eye drops as stated above, an injection of $0.1-0.2 \mathrm{ml}$ (2.5 mg to $5 \mathrm{mg} 5 \mathrm{FU})$ was injected in the belly of the pterygium with a 27 gauge needle. An average of 3.3 injections (range 1-8) was needed [11]. This was done at detection of conjunctival recurrence of pterygium [11], avoiding repeat surgery, which can be difficult as harvesting another conjunctival autograft can be difficult if one has previously been done.

\section{Statistical analysis}

Statistical analysis was performed by using GraphPad Prism 7.03 (GraphPad Software La Jolla, CA, USA). Wilcoxon ad hoc test was used to analyse the data for pterygium length and OCT thickness while paired $t$-test was used to analyse data for clinical grading.

\section{Laboratory study}

Four patients returned for excision of pterygium for cosmetic reasons, 5 months after last injection. Pterygium tissue from these patients and from three non-injected controls matched for severity (controls) were subjected to immunohistological examination. Pterygium tissue was fixed in $4 \%$ paraformaldehyde for $2 \mathrm{~h}$ and embedded in paraffin. Thin sections $(4 \mu \mathrm{m})$ were cut, deparaffinized and antigen retrieval carried out by incubating slides in $10 \mathrm{mM}$ sodium citrate solution, $\mathrm{pH}$ 6.0 (IHC antigen retrieval solution-low pH; Thermo Fisher Scientific, UK) for $15 \mathrm{~min}$ at $60^{\circ} \mathrm{C}$. The slides were then incubated with $5 \%$ normal donkey serum in $0.03 \%$ Triton$\mathrm{X} 100$ for $1 \mathrm{~h}$ to block any non-specific binding sites. Sections were stained with specific primary antibodies (Supplementary Table 1) or negative control (no primary or normal mouse IgG1) overnight at $4{ }^{\circ} \mathrm{C}$ followed by secondary antibody staining (Alexa Fluor 488 or 594 conjugated donkey antirabbit IgG or donkey anti-mouse IgG, Thermo Fisher scientific) at room temperature for $1 \mathrm{~h}$. The stained sections were mounted in DAKO fluorescent mounting media and examined using fluorescent microscope (B51X Olympus, Japan) and processed with Adobe Photoshop CS4 (Adobe Systems, CA). Histological sections in duplicate, from each of the three control samples and four injected pterygia were used for each of the seven antibodies indicated in Supplementary Table 1.

\section{Results}

A total of 16 eyes of 16 patients received 5FU and Avastin injections. Of the sixteen patients, seven were females (44\%), and nine were males (56\%). Mean age was 54 years (range was from 24 to73 years). Fourteen patients received five injections, one received three injections and one received two injections. All patients experienced stinging during injection of Avastin but not with 5FU. All patients showed subconjunctival and/or intra-lesional haemorrhage after injection (Supplementary Fig. 1), which resolved in 2 weeks.

All patients showed arrest of progression over the followup period. There was a mean reduction of $0.10 \mathrm{~mm} \pm 0.1$ in pterygium length in $56 \%$ of the patients while the rest did not show any change $(P<0.005$, median $=0.041,95 \%$ confidence interval $(\mathrm{CI})=0.024-0.177)$. Twenty-two percent of the patients showed a reduction in astigmatism (mean magnitude of difference vector was 10.1 ), $11 \%$ showed no difference (mean magnitude of difference vector was 1 ) while $67 \%$ had increased astigmatism (mean magnitude of difference vector was 5) following the injections. The mean magnitude of difference vector was 5.7 and the range of astigmatic change was from 0.8 to19.4. Eighty-nine percent of patients had reduced thickness of pterygium as evidenced by difference in OCT measurements while the rest had no change. The mean reduction in thickness was $35.8 \mu \mathrm{m} \pm 31.29,(P<0.001$, median $=29 \mu \mathrm{m}, 95 \% \mathrm{CI}=11.7-59.9$ ).

The clinical grade of the pterygium had significantly reduced in $62.5 \%$ while it remained the same in $37.5 \%$. No patient showed an increase in clinical grade of pterygium. Ninety percent of the patients who showed reduction of clinical grade had one clinical grade improvement while $10 \%$ had a two clinical grades improvement (Supplementary Fig. 2, Figs. 1-2) (Mean pre-injection grade was 2.313, mean post-injection grade was $1.625, P<0.001,95 \% \mathrm{CI}=$ $0.367-1.008)$. Two patients developed superficial punctate keratitis after the fourth and fifth injection. This resolved on cessation of injection.

\section{Laboratory study}

Atypical fibroblast cells and vascular endothelium showed positive staining for bFGF in control pterygium samples (Fig. 3a), whereas in injected pterygium (Fig. 3b) weak bFGF immunoreactivity was noted only at the basal aspect of vascular endothelium. Monoclonal antibody against VEGF showed positive immunoreactivity in 
control pterygium samples (Fig. 3c) and a significantly reduced staining in injected samples (Fig. 3d). EGF was abundantly present in pterygium epithelium and around basal aspects of vascular endothelium of control samples (Fig. 3e), but in injected samples it was only detected at low levels around basal aspect of vascular endothelium (Fig. 3f). Reactivity of collagen-I was moderately reduced
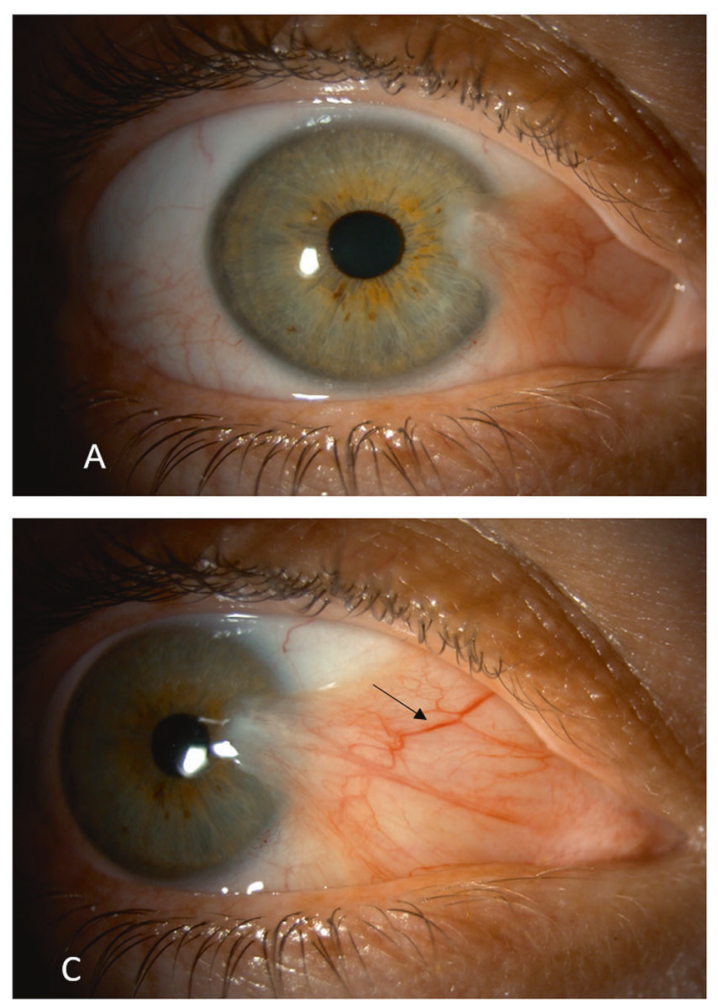

Fig. 1 Representative images of a pterygium before and after three injections for patient 2 (slit lamp image, diffuse illumination). a, c Preinjection images of pterygium with patient looking straight (a) and temporally (c). The lesion appears fleshy with dilated and full vessels

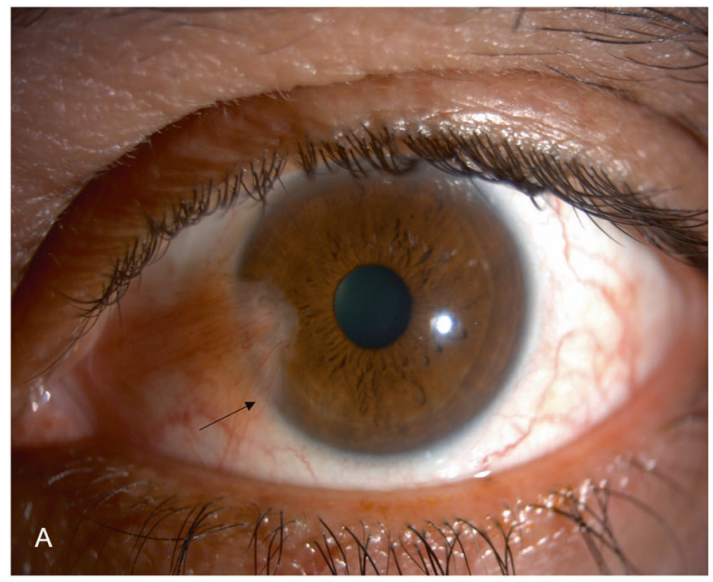

Fig. 2 Representative images of a pterygium before and after five injections for patient 3 (slit lamp image, diffuse illumination). a The pterygium before injections is fleshy and vascular obscuring the in injected samples (Fig. 3h) compared to control samples (Fig. 3g). Secreted protein acidic and cysteine rich (SPARC) was shown to be positively expressed in all control samples but unlike bFGF, it remained unchanged in sections of injected samples (Fig. 4). Antibodies against von-Willebrand factor (vWF) were used to detect blood vessels, and against lymphatic vessel endothelial
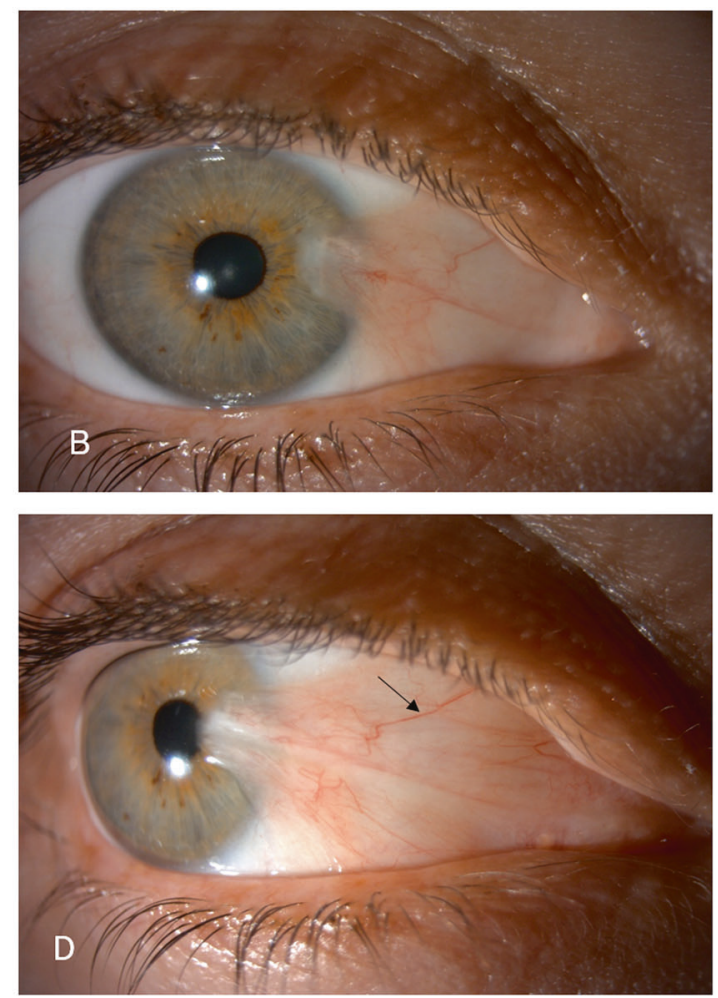

(arrow). b, d Post-injection images show reduction in vascularity with patient looking straight (b) and temporally (d). The arrow in $\mathbf{d}$ points to a vessel that has attenuated with barely visible branches

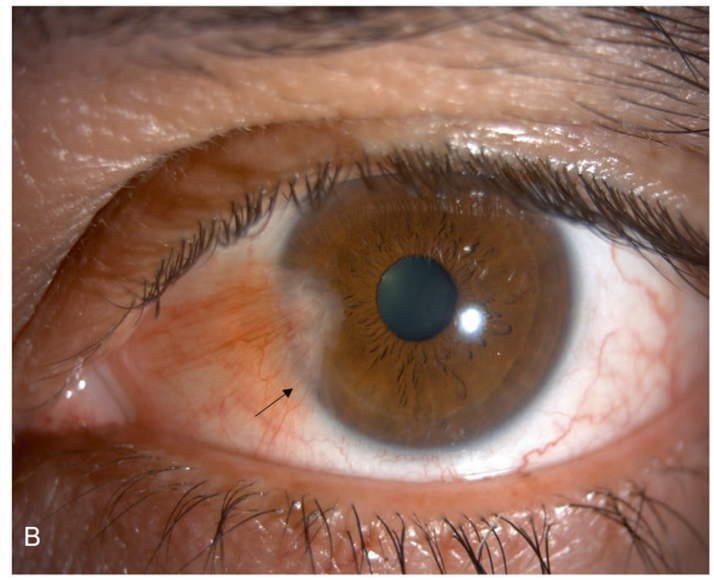

underlying limbus (arrow). b The pterygium after injections is atrophic as evidenced by increased visibility of the outline of the underlying limbus especially inferiorly (arrow) 
Fig. 3 Immunofluorescence analysis of control and injected pterygium samples.

Representative sections of sample from control $(\mathbf{a}-\mathbf{g} ; n=3)$ and injected $(\mathbf{b}-\mathbf{h} ; n=4)$ groups. Positive

immunoreactivity for bFGF in fibroblast cells (a); VEGF in vascular endothelium and extracellular matrix (c), EGF in epithelial cells (e) and Collagen$\mathrm{I}$ in vascular basement membrane (g) are demonstrated in the control sections. Reduced or weakened staining for these proteins is seen in corresponding images post injection (b-h). Scale bar $=50 \mu \mathrm{m}(\times 400)$
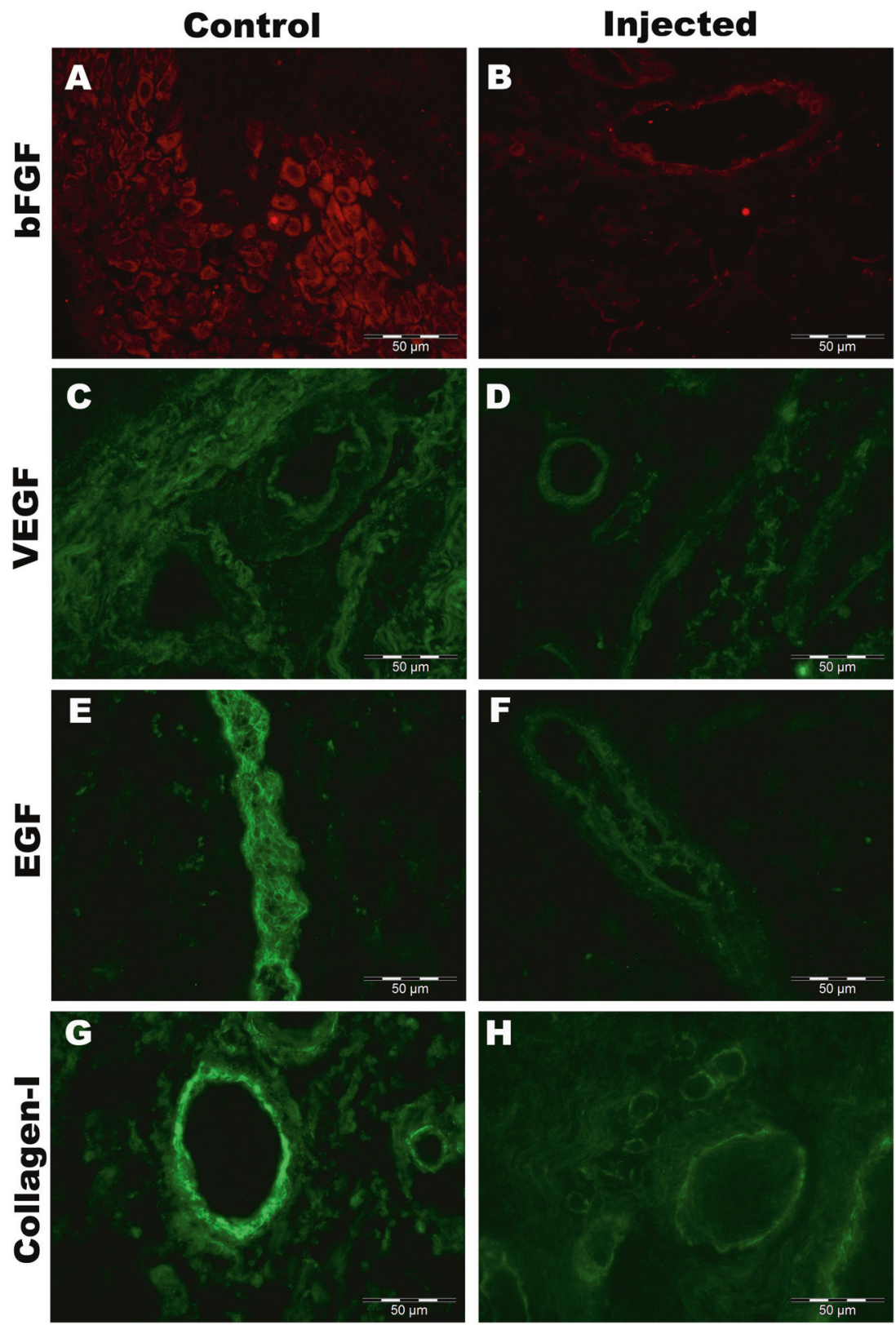

hyaluronan receptor (LYVE-1) to detect lymphatic vessels. Some vascular structures showed dual staining for both $\mathrm{VWF}$ and LYVE-1 in sections of control samples (Fig. 5) whereas reduced expression was noted in injected samples. This amounted to $64.3 \%$ reduction in vWF staining and $61.9 \%$ in LYVE-1 stained vasculature after injection (Supplementary Table 2).

\section{Discussion}

Recurrence of pterygium is the major complication after surgical excision of the lesion. The two main pathological processes in pterygium are fibroblast proliferation and angiogenesis. Many studies have demonstrated that the pathogenesis and recurrence of pterygia can be attributed to the increased expression of molecules such as VEGF, transforming growth factor, platelet derived growth factor and bFGF [17]. VEGF was found to be highly increased in pterygia and is believed to be involved directly or indirectly in formation of pterygia [18].

Intervention to reduce incidence of recurrence following surgery for primary or recurrent pterygia include the use of autologous conjunctival graft and application of antimetabolites either 5FU or MMC intraoperatively. Intralesion injection of antimetabolites and anti-VEGF drugs have also been used to treat recurrent pterygium [11-14]. Encouraged by the beneficial effects of treating recurrence, 

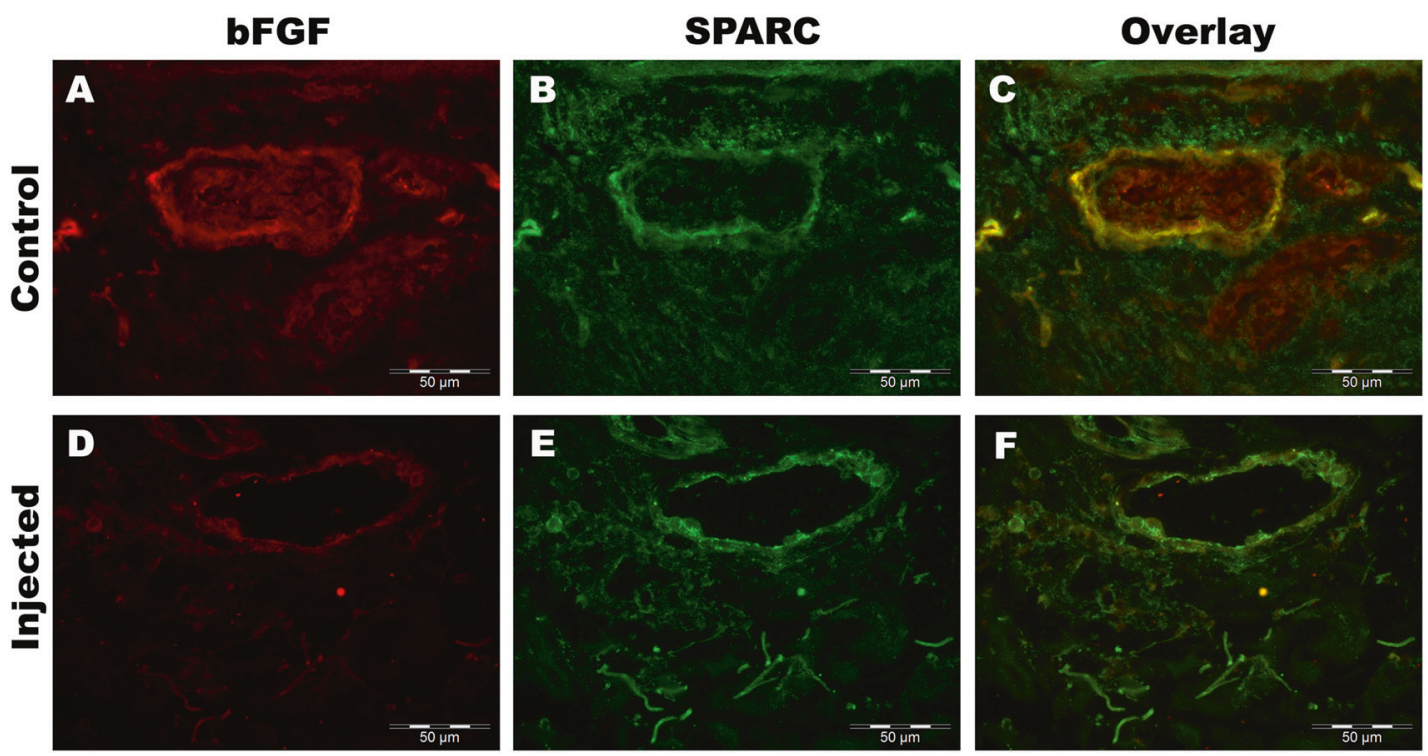

Fig. 4 Double labelling of bFGF and SPARC. Representative sections of sample from control $(n=3)$ and injected $(n=4)$ groups. Labelling for bFGF is demonstrated by red fluorescence $(\mathbf{a}, \mathbf{d})$ and SPARC by green fluorescence $(\mathbf{b}, \mathbf{e})$ in multiple sections of the same pterygium

vessel in control and injected samples. Overlay micrographs indicate double labelling for bFGF and SPARC (orange/yellow fluorescence; c, f). The staining for bFGF is markedly reduced in the injected sample but that of SPARC is unchanged. Scale bar $=50 \mu \mathrm{m}(\times 400)$
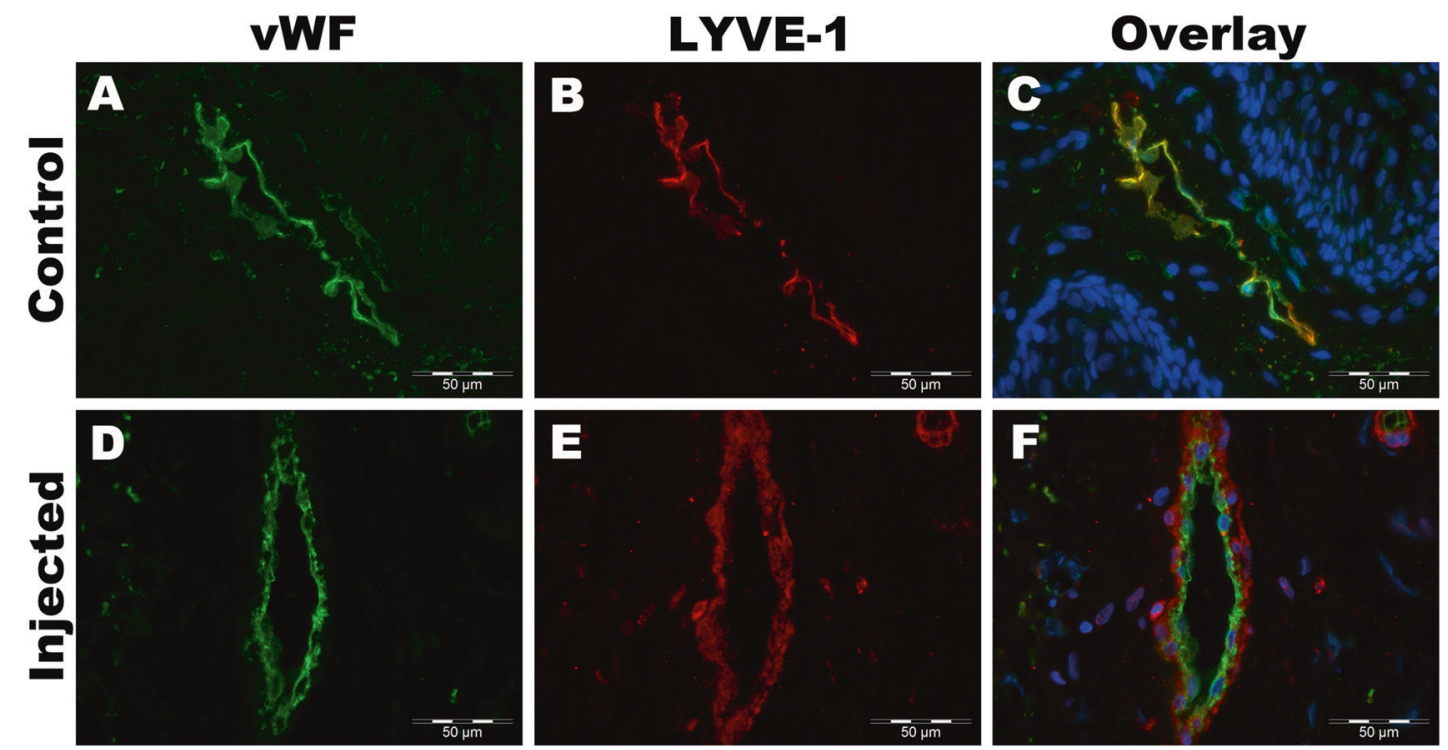

Fig. 5 Co-localisation of vWF (vascular) and LVYE-1 (lymphatic) channels. Representative micrographs of control $(n=3)$ and injected $(n=4)$ samples. vWF is indicated in green $(\mathbf{a}, \mathbf{d})$ and LYVE-1 in red

both agents have also been used individually in treatment of primary pterygium with considerable success [8]. 5FU inhibits DNA synthesis and proliferation of fibroblasts [19]. Anti-VEGF antibodies bind to isoforms of VEGF and inhibit the VEGF-receptor interaction preventing angiogenesis [13].

In our study we injected both agents together, for the treatment of primary pterygium. We found that this approach resulted in reduction in the clinical grade, (b, e). Overlay micrograph of control section (c) indicates colocalisation of vWF and LYVE-1. In injected sample (f), LYVE-1 stained cells surround vWF stained endothelium

thickness and vascularity of the pterygium. This twopronged approach addressing both the main pathological processes may work synergistically affecting thickness and vascularity of the pterygium. Some patients also showed a slight reduction in the length of the pterygium. This is most likely due to the reduction in thickness at the apex, but in any case, was less than $0.2 \mathrm{~mm}$ and clinically insignificant. Significantly, the progression of pterygium was arrested in all cases and the cosmesis too had improved in $62.5 \%$ with 
$56 \%$ of the concerned patients noticing the difference subjectively. Some of these individuals are unlikely to proceed to surgery making the study clinically more relevant. Interestingly, however, the astigmatism increased in $67 \%$ of patients post injection. This could be related to the retraction of the fibrotic tissue post injection exerting a greater force on the cornea inducing further deformation. Malik et al. [12] compared the mean change in corneal astigmatism and clinical appearance after intralesional injection of $5 \mathrm{FU}$ in primary and recurrent pterygia. They treated all patients with $0.1 \mathrm{ml}$ of $5 \mathrm{FU}(5 \mathrm{mg})$ weekly injections for 4 weeks and demonstrated a reduction of the astigmatism. However, Singh et al. [16] use only Avastin injection and showed not difference in astigmatism. Unlike these studies, we injected both 5FU and Avastin, which could account for the increased astigmatism we noted, probably as a consequence of fibrous tissue contraction. Most studies that have used either 5FU or Avastin [15-17] concluded that improvements in appearance, colour and surface area of pterygium could be safely achieved.

Despite anaesthesia, all patients complained of a stinging sensation during injection of Avastin but not with 5FU. Hence, we injected the latter before the former. Avastin solution is acidic (pH 6.13) and 5FU is alkaline ( $\mathrm{pH} 8.89)$, which could explain the difference in patient experience. Apart from mild punctate keratitis, no adverse events were recorded. Post-injection, four patients requested surgery to remove the residual pterygium. Intraoperative bleeding was much less compared to previous experience with uninjected lesions. This is an anecdotal observation as the study was not designed to pick up this difference. These four samples were examined by immunohistology.

\section{Immunohistology}

Immunohistochemical studies have shown increased expression of VEGF in pterygium compared to normal conjunctiva $[8,18]$. VEGF receptors 2 and 3 are also increased in pterygium [8]. Mohamed et al. [19] conducted a randomised control study with one group receiving a single Avastin injection while the other did not receive any. Similar to our results, they found a reduced mean vessel count and a significant reduction in VEGF expression in injected pterygium samples. vWF is one of the proteins involved in blood vessel function. It has been demonstrated in the pterygial blood vessel walls and could be involved in pterygial angiogenesis [20]. Lymphatic vessel endothelial cells express specific proteins including LYVE-1 [21]. Our study demonstrated that there was a reduction in both vWF and LYVE-1 in the injected samples indicating decreased angiogenesis and lymphangiogenesis, which is a novel finding. Interestingly, in control pterygium samples there was a close localisation of both vWF and LYVE-1 in relation some vessels, which could be related to immature vessels or proximity of mononuclear cells [22].

Detorakis et al. [23] found that levels of bFGF were higher in pterygium than normal conjunctiva. Collagen types I, III and IV were found in normal conjunctiva and pterygium [24]. In our injected samples both bFGF and Collagen-I were reduced, which is more likely to be due to the effect of $5 \mathrm{FU}$ as it primarily targets the proliferation of fibroblasts. SPARC is a matrix protein which has several roles in wound remodelling and modification of collagen production as well as in angiogenesis and high levels have been reported in pterygium [8]. We were not able to demonstrate any effect of our injections on the SPARC expression despite reduction in vascularity and collagen suggesting that bFGF plays a greater role in the pathophysiology of pterygium. Pterygium epithelium and fibroblasts derived from pterygia also express epidermal growth factor receptors [25]. We detected EGF at the basal aspect of pterygium vascular endothelium, which was reduced after injections suggesting that EGF also contributed to angiogenesis in pterygium.

This study demonstrates that proliferative activity in pterygium and its progression can be modified by drugs targeting the various growth factors and cells involved in the pathogenesis of pterygium. Such a medical approach in the management of pterygium, the treatment of which is essentially surgical, is a viable option to consider for both recurrent and primary pterygia.

\section{Summary}

\section{What was known before}

- The only viable option for management of pterygium is surgical excision.

\section{What this study adds}

- Combined 5FU and Avastin injections are a viable option in arresting progress of primary pterygium. They act synergistically to affect collagen, fibroblasts, vascular and lymphatic vessels.

Funding This study was funded by a small grant award from 'Fight for Sight', reference: 1708/09. The funding organisation had no role in the design or conduct of this research.

\section{Compliance with ethical standards}

Conflict of interest The authors declare that they have no conflict of interest. 
Publisher's note: Springer Nature remains neutral with regard to jurisdictional claims in published maps and institutional affiliations.

\section{References}

1. Kim KW, Park SH, Kim JC. Fibroblast biology in pterygia. Exp Eye Res. 2016;142:32-9.

2. Detorakis ET, Spandidos DA. Pathogenetic mechanisms and treatment options for ophthalmic pterygium: trends and perspectives (Review). Int J Mol Med. 2009;23:439-47.

3. Teng CC, Patel NN, Jacobson L. Effect of subconjunctival bevacizumab on primary pterygium. Cornea. 2009;28:468-70.

4. Mauro J, Foster CS. Pterygia: pathogenesis and the role of subconjunctival bevacizumab in treatment. Semin Ophthalmol. 2009;24:130-4.

5. Di Girolamo N, Chui J, Coroneo MT, Wakefield D. Pathogenesis of pterygia: role of cytokines, growth factors, and matrix metalloproteinases. Prog Retin Eye Res. 2004;23:195-228.

6. Gumus K, Karakucuk S, Mirza GE, Akgun H, Arda H, Oner AO. Overexpression of vascular endothelial growth factor receptor 2 in pterygia may have a predictive value for a higher postoperative recurrence rate. Br J Ophthalmol. 2014;98:796-800.

7. Ling $\mathrm{S}$, Li Q, Lin $\mathrm{H}$, Li W, Wang T, Ye H, et al. Comparative evaluation of lymphatic vessels in primary versus recurrent pterygium. Eye. 2012;26:1451-8.

8. Bornstein P, Sage EH. Matricellular proteins: extracellular modulators of cell function. Curr Opin Cell Biol. 2002;14:608-16.

9. Riordan-Eva P, Kielhorn I, Ficker LA, Steele AD, Kirkness CM. Conjunctival autografting in the surgical management of pterygium. Eye. 1993;7:634-8.

10. Kheirkhah A, Hashemi H, Adelpour M, Nikdel M, Rajabi MB, Behrouz MJ. Randomized trial of pterygium surgery with mitomycin $\mathrm{C}$ application using conjunctival autograft versus conjunctival-limbal autograft. Ophthalmology. 2012;119:227-32.

11. Said DG, Faraj LA, Elalfy MS, Yeung A, Miri A, Fares U, et al. Intra-lesional 5 fluorouracil for the management of recurrent pterygium. Eye. 2013;27:1123-9.

12. Malik S, Khan MS, Basit I. Comparison of primary versus recurrent pterygium after intralesional 5-Fluorouracil. J Pak Med Assoc. 2016;66:559-62.

13. Bayar SA, Kucukerdonmez C, Oner O, Akova YA. Subconjunctival bevacizumab in the impending recurrent pterygia. Int Ophthalmol. 2014;34:541-7.
14. Nava-Castañeda A, Ulloa-Orozco I, Garnica-Hayashi L, HernandezOrgaz J, Jimenez Martinez MC, Garfias Y. Triple subconjunctival bevacizumab injection for early corneal recurrent pterygium: one-year follow-up. J Ocul Pharmacol Ther. 2015;31:106-13.

15. Khan MS, Malik S, Basit I. Effect of intralesional 5 fluorouracil injection in primary pterygium. Pak J Med Sci. 2016;32:130-3.

16. Singh P, Sarkar L, Sethi HS, Gupta VS. A randomized controlled prospective study to assess the role of subconjunctival bevacizumab in primary pterygium surgery in Indian patients. Indian J Ophthalmol. 2015;63:779-84.

17. Besharati MR, Manaviat MR, Souzani A. Subconjunctival bevacizumab injection in treatment of pterygium. Acta Med Iran. 2011;49:179-83.

18. Lee JK, Song YS, Ha HS, Park JH, Kim MK, Park AJ. Endothelial progenitor cells in pterygium pathogenesis. Eye. 2007;21:1186-93.

19. Mohamed TA, Soliman W, Fathalla AM, El Refaie A. Effect of single subconjunctival injection of bevacizumab on primary pterygium: clinical, histopathological and immunohistochemical study. Int J Ophthalmol. 2018;11:797-801.

20. Naib-Majani W, Eltohami I, Wernert N, Watts W, Tschesche H, Pleyer U. Distribution of extracellular matrix proteins in pterygia: an immunohistochemical study. Graefes Arch Clin Exp Ophthalmol. 2004;242:332-8.

21. Cursiefen C, Schlötzer-Schrehardt U, Küchle M, BreitenederGeleff S, Alitalo K, Jackson D. Lymphatic vessels in vascularized human corneas: immunohistochemical investigation using LYVE-1 and podoplanin. Invest Ophthalmol Vis Sci. 2002;43:2127-35.

22. Schlereth SL, Neuser B, Caramoy A, Grajewski RS, Koch KR, Schrödl F, et al. Enrichment of lymphatic vessel endothelial hyaluronan receptor 1 (LYVE1)-positive macrophages around blood vessels in the normal human sclera. Invest Ophthalmol Vis Sci. 2014;55:865-72.

23. Detorakis ET, Zaravinos A, Spandidos DA. Growth factor expression in ophthalmic pterygia and normal conjunctiva. Int $\mathrm{J}$ Mol Med. 2010;25:513-6.

24. Dake Y, Mukae R, Soda Y, Kaneko M, Amemiya T. Immunohistochemical localization of collagen types I, II, III, and IV in pterygium tissues. Acta Histochem. 1989;87:71-4.

25. Kase S, Osaki M, Sato I, Takahashi S, Nakanishi K, Yoshida K, et al. Immunolocalisation of E-cadherin and beta-catenin in human pterygium. Br J Ophthalmol. 2007;91:1209-12. 